\title{
THE TESLA FREE ELECTRON LASER
}

\author{
J. Rossbach, for the TESLA FEL collaboration \\ DESY, Notkestrasse 85, D22603 Hamburg, Germany
}

\section{Abstract}

The TESLA Free Electron Laser (FEL) makes use of the high electron beam quality that can be provided by the superconducting TESLA linac to drive a single pass FEL at wavelengths far below the visible. To reach a wavelength of 6 nanometers, the TESLA Test Facility (TTF) currently under construction at DESY will be extended to $1 \mathrm{GeV}$ beam energy. Because there are no mirrors and seed-lasers in this wavelength regime, the principle of Self-AmplifiedSpontaneous-Emission (SASE) will be employed. A first test of both the principle and technical components is foreseen at a photon wavelength larger than 42 nanometers.

With respect to linac technology, the key prerequisite for such single-pass, high-gain FELs is a high intensity, diffraction limited, electron beam to be generated and accelerated without degradation. Key components are RF guns with photocathodes, bunch compressors, and undulators. The status of design and construction as well as both electron and photon beam properties are discussed.

Once proven in the micrometer to nanometer regime, the SASE FEL scheme is considered applicable down to Angstrom wavelengths. This latter option is particularly of interest in context with the construction of a linear collider, which requires very similar beam parameters. The status of conceptual design work on a coherent X-ray user facility integrated into the TESLA Linear Collider design is briefly sketched.

\section{INTRODUCTION}

Most of the information humans receive from their environment are provided by photons. Thus it is not surprising that also for research in natural sciences photons, ranging from radio frequencies to hard $\gamma$-rays, provide the most important tool to study nature. For the photon range beyond the narrow optical spectral window up to the hundred $\mathrm{keV}$ $\gamma$-regime synchrotron radiation has provided over the past thirty years an increase in flux and brightness by more than ten orders of magnitude. The development of storage ring designs with special magnetic components, called wigglers and undulators, over the past twenty years has led to third generation machines specially designed for synchrotron radiation research.

Most recent successes in accelerator development concerning linear accelerators have opened the route to a new jump in photon source quality. X-ray lasers coupled to linear accelerators can possibly provide coherent X-rays with true laser properties through very long undulators. Although such a source of coherent laser-like X-rays would have many applications and the search for such a device has occupied many scientists, a practical solution to this problem has not yet been realized. To be an efficient research tool, such a source would have to provide stable intensities with short pulses and repetition frequencies similar to what is found in optical lasers. Exactly this seems to be possible by using the so-called self amplified spontaneous emission process SASE.

The basic principle [1] makes use of the fact that an electron beam of sufficient quality, passing a long undulator magnet, exponentially amplifies an initially existing radiation field, if the photon wavelength $\lambda_{p h}$ matches a resonance condition determined by undulator parameters and the beam energy:

$$
\lambda_{p h}=\frac{\lambda_{u}}{2 \gamma^{2}}\left(1+K^{2}\right)
$$

Here, $\gamma$ is the electron energy in units of its rest mass, $\lambda_{u}$ is the undulator period, $\mathrm{K}$ is the undulator parameter, and a helical undulator is assumed. Coherent, stimulated emission is achieved because a bunch density modulation of the electron beam at the optical wavelength builds up during the passage of the bunch through the undulator.

If the desired wavelength is very short, one can, instead of providing the "initially existing radiation field" by a conventional laser, consider the undulator radiation radiated spontaneously in the first part of the undulator as an input signal, see Fig. 1. This principle of Self-Amplified-

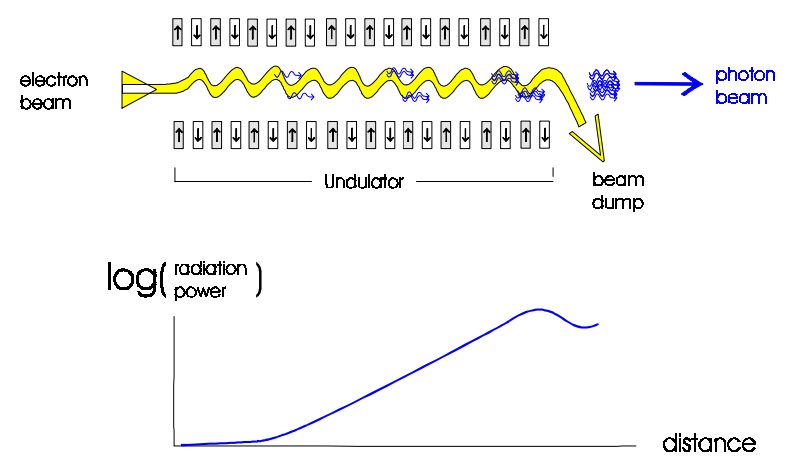

Figure 1: Schematic Diagram of a Single-Pass Free Electron Laser (FEL) operating in the Self-AmplifiedSpontaneous-Emission (SASE) mode.

Spontaneous-Emission (=SASE) [2], [3] also does not require the optical cavity resonator normally used in multipass, longer wavelength FELs and can hence, in principle, deliver light with wavelength in the Angstrom regime. Compared to state-of-the-art synchrotron radiation sources, one expects a transversely fully coherent beam, larger average brilliance, and, in particular, a pulse length of about 


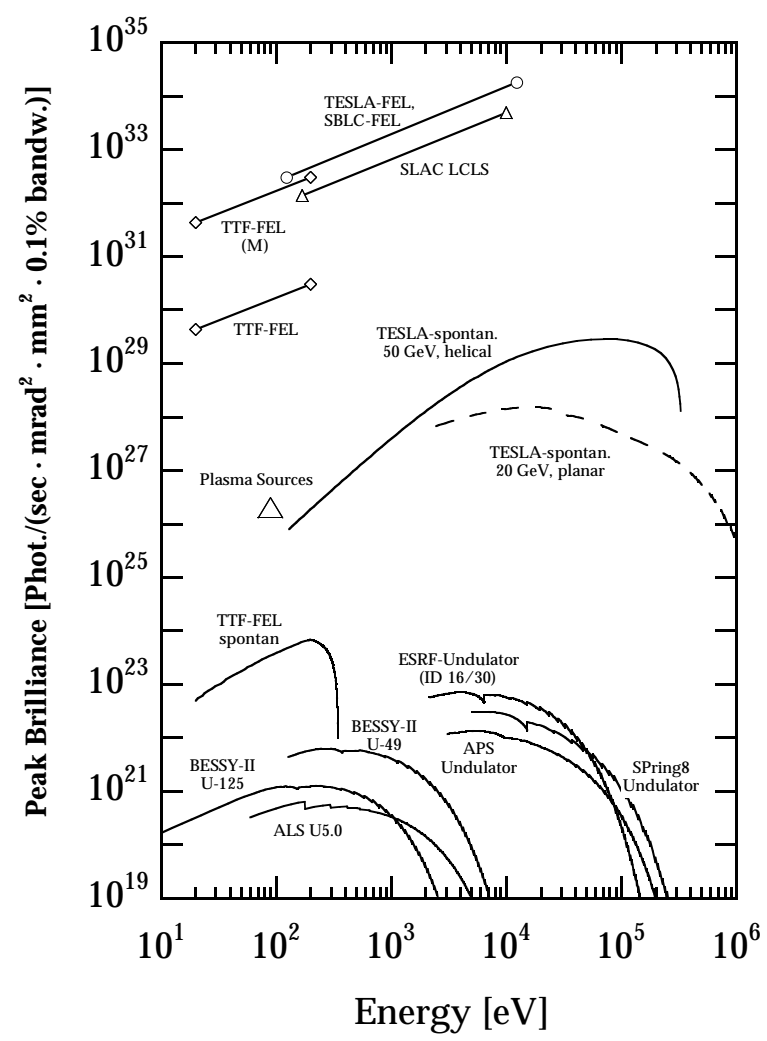

Figure 2: Spectral peak brilliance of X-ray FELs based on SBLC and TESLA linear colliders, together with that of third generation synchrotron radiation sources and the LCLS Free Electron Laser project discussed at SLAC, Stanford, USA [8]. For comparison, also the spontaneous spectra of two XFEL undulators are shown. The peak brilliance of state-of-the-art plasma lasers is also noted.

200 fs FWHM with eight or more orders of magnitude larger peak brilliance, see Figs. 2 and 3. The peak power is in the range $1-100 \mathrm{GW}$. The average brilliance is significantly larger than for the LCLS device proposed earlier[8] mainly because TESLA accelerates a bunch train of $\sim 10000$ bunches at a rate of $5 \mathrm{~Hz}$, i.e. the mean repetition rate is much higher. As the SASE FEL is completely independent of atomic excitation levels, it can be tuned over a wide range of wavelengths.

If, according to technical restrictions, undulator periods in the order of few centimeters are assumed, Eq.1 indicates that multi-GeV electron energies are required to achieve photon wavelengths in the Angstrom regime. This goes in parallel with the large energy gain needed in order to sufficiently reduce the beam emittance provided by the injector. Therefore, the electron accelerator required for an X-ray FEL will be a major investment. Also, construction, commissioning and operation of such a device needs a considerable staff of well-trained experts. Last but not least, a large laboratory site will be needed to accommodate the various

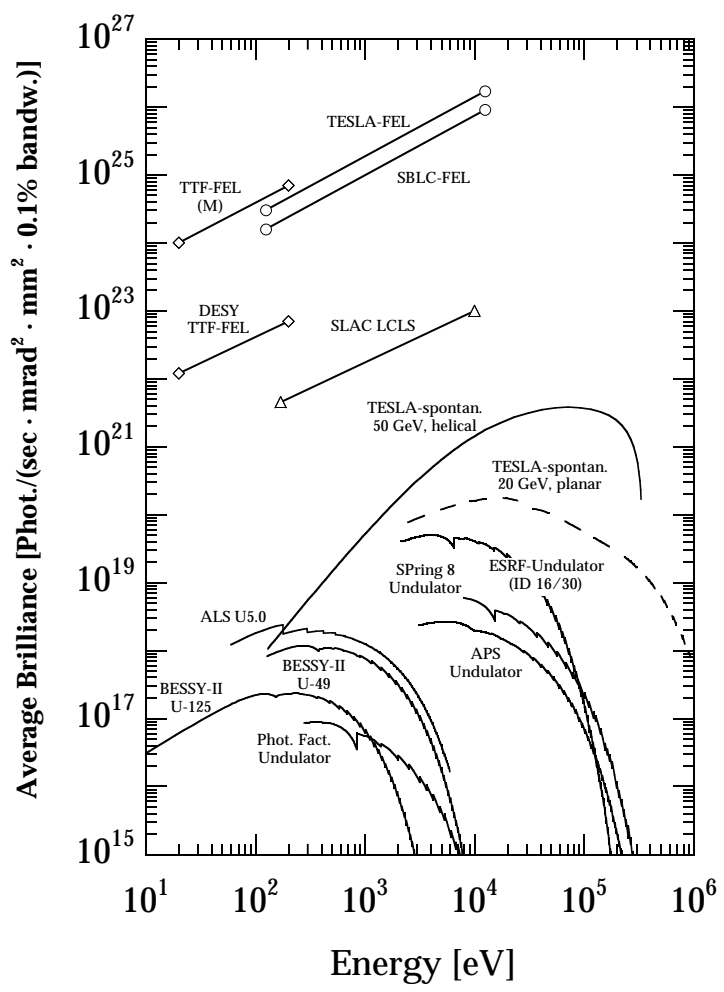

Figure 3: See Fig. 2, but spectral average brilliance values are shown.

user facilities. It was concluded, that it will be most attractive to combine an X-ray FEL facility into a linear collider installation [4], and that this will be possible without serious mutual interference.

\section{THE TESLA CONCEPT}

The different linac concepts considered by linear collider study groups mainly differ with respect to the choice of RF frequency [5]. Roughly speaking, higher RF frequencies offer a higher accelerating gradient, i.e. a shorter tunnel length, at the price of reduced power efficiency and larger beam energy spread. In contrast, low frequency linacs (down to $1.3 \mathrm{GHz}$ for the superconducting TESLA linac) promise very good beam quality, because each electron bunch extracts only a small fraction of the large energy stored in the big cavity volume (small wakefield effects, see section 5). For an X-ray FEL, the highest priority is electron beam quality and large average beam current, while high accelerating gradient might be of minor importance. Finally, it is attractive for many users if a large variability in pulse timing can be offered. In this respect, the TESLA linac again offers an advantage, because a large variety of timing patterns can be realised due to the very large duty cycle of $1 \%$. Thus, for FELs there is a clear preference for low frequency linacs. A Conceptual Design Report on such a combined laboratory has been worked out by the international TESLA collaboration [9]. This paper also discusses 
a normal-conducting linac version based on conventional S-band technology $(3 \mathrm{GHz})$, called SBLC.

It is understood that the challenging goal of an $1 \AA$ FEL cannot be achieved within a single step. Thus, a SASE proof-of-principle experiment will be installed at the TESLA Test Facility (TTF), called TTF FEL Phase 1, see Fig. 4. Based on three TESLA accelerating modules, 390 $\mathrm{MeV}$ beam energy will allow to reach a photon wavelength of $\lambda_{p h}=42 \mathrm{~nm}$. First operation is scheduled for 1999. A more detailed description and the status is given in Ref. [6].

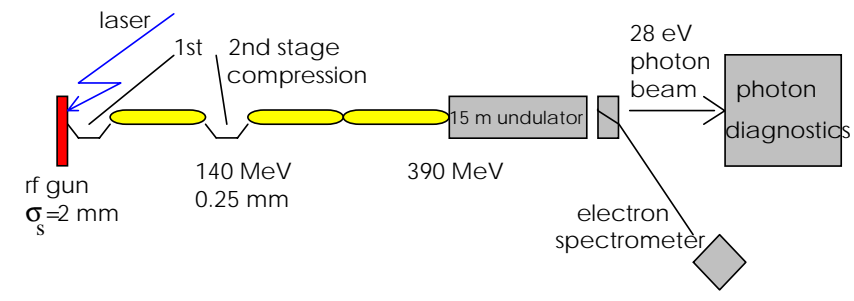

Figure 4: Schematic diagram of the TESLA Test Facility installation including the Phase 1 SASE FEL components.

As a second phase, an energy upgrade to $1 \mathrm{GeV}$ has been approved which will bring the wavelength down to $6 \mathrm{~nm}$ in the first harmonic. Besides testing SASE in the soft X-ray regime, an attractive physics program has been identified for this installation [7], covering applications ranging from magnetic materials research and various pump-and-probe experiments to photochemistry and X-ray microscopy of biological samples. In the following sections, design and status of key TTF FEL components are discussed.

\section{ELECTRON SOURCE}

The transverse coherence condition imposes a tight requirement on the transverse emittance $\varepsilon_{t}$ of the electron beam [10]:

$$
\varepsilon_{t}^{n} \leq \frac{\gamma \lambda_{p h}}{4 \pi}
$$

$\varepsilon_{t}^{n}$ is the normalized emittance. For $\lambda_{p h}=6 \mathrm{~nm}, \gamma=2000$, Eq. 2 requires $\varepsilon_{t}^{n}<1 \pi \mathrm{mm}$ mrad. Actually, this condition is not very strict, but the saturation length significantly increases if $\varepsilon_{t}^{n}$ is larger. Thus, we aim at $\varepsilon_{t}^{n}=1 \pi \mathrm{mm} \operatorname{mrad}$ for the rms electron emittance of a $1 \mathrm{nC}$ bunch charge from an RF electron gun [11], and we allow for a factor of 2 in emittance dilution during longitudinal bunch compression and acceleration up to $1 \mathrm{GeV}$, see Table 1 .

Compared to state-of-the-art, space-charge compensated, RF photoinjectors [12], the TESLA gun has to provide a longer bunch train and smaller emittance. The laser developed [13] provides an intensity stability of a few \% and will be delivered to DESY in summer 1997. Neodymium doped Yttrium-Lithium Fluorid (Nd:YLF) has been chosen as the active medium since it combines a small thermal lensing effect with a high induced emission cross section and a relatively long fluorescence lifetime (480 $\mu \mathrm{s})$. A pulse train with the desired spacing is selected from the bunches in a pulse train oscillator working at $36 \mathrm{MHz}$ by means of Pockels cells. The light pulses are amplified in a linear low gain amplifier chain and then quadrupled in frequency. Relay imaging techniques in combination with spatial filtering is used to produce a radially uniform intensity profile.

To gain in emittance, any asymmetries in the RF field have been avoided in the design. Since previous calculations have revealed an emittance contribution of more than $1 \pi \mathrm{mrad} \mathrm{mm}$ for a conventional asymmetric input coupler, a symmetric input coupler has been developed. Fig. 5 shows a schematic layout of the gun and the coupler. At present, most components have been built or are under construction. An Aluminum model has successfully been tested to verify the coupler and cavity geometry.

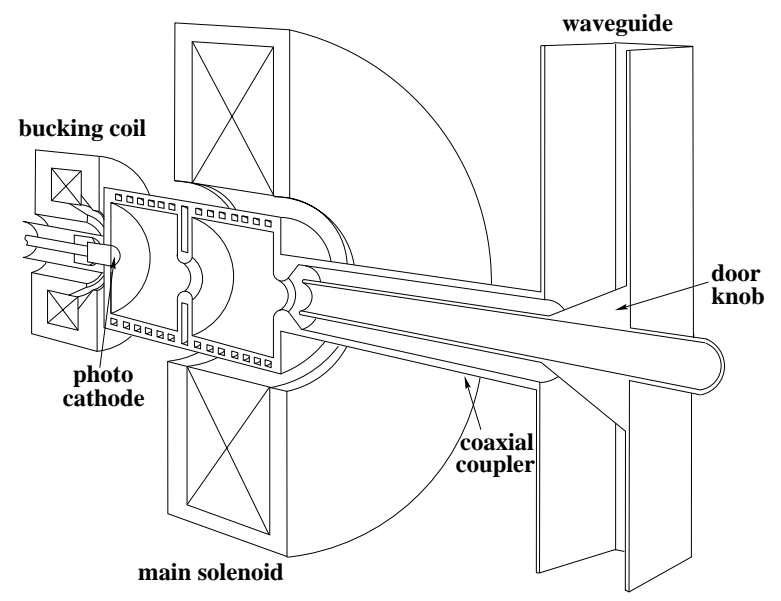

Figure 5: Cross sectional view of the RF gun (schematic) with the gun cavity, the cathode plug, the main solenoid and the bucking coil, and the coaxial input coupler. The RF is coupled from the rectangular waveguide via a door knob transition into the coaxial line and the cavity. The electrons leave the gun through the inner conductor.

\section{BUNCH COMPRESSION}

In order to achieve laser saturation within a single passage, XFELs require electron pulse currents of few kiloamperes. Because of space charge effects, such high currents cannot be generated directly from the RF gun cathode without blowing up the transverse emittance. Instead, a 7 ps long, 120 A beam is produced, accelerated, and longitudinally compressed by a factor of 20 . This compression takes place in several steps at different energies. Bunch compressors are beamline sections which longitudinally compress the bunch using path length differences in a magnetic chicane. This is a well established technique, but has not yet been realized with extremely brilliant electron beams. Although simple in first-order theory, the physics of bunch compression becomes very challenging if collective effects like space charge forces [14],[15] and wakefields (see next section) are taken into account. Especially emittance conservation is then a critical issue [16]. 
Table 1: Parameters of the FEL at the TESLA Test Facility (TTF) (phases 1 and 2) and at the TESLA Linear Collider.

\begin{tabular}{|l|c|c|c|c|}
\hline Linac Parameters & Unit & TTF FEL Phase 1 & TTF FEL Phase 2 & TESLA X-ray FEL \\
\hline gradient for XFEL operation & $\mathrm{MV} / \mathrm{m}$ & $10-15$ & $10-15$ & $10-25$ \\
linac repetition rate $f_{\text {rep }}$ for XFEL & $\mathrm{Hz}$ & 10 & 10 & 5 \\
bunch length (rms) & $\mathrm{fs}$ & 800 & 160 & 80 \\
bunch spacing & $\mathrm{ns}$ & 93 & 93 & 93 \\
number of bunches per train & & 11315 & 11315 & 11315 \\
bunch train length & $\mu \mathrm{s}$ & 1050 & 1050 & 1050 \\
bunch charge & $\mathrm{nC}$ & 1 & 1 & 1 \\
normalized emittance at undul. entrance & $\pi \mathrm{mrad} \mathrm{mm}$ & 2 & 2 & 1 \\
longitudinal emittance & $\mathrm{keV} \mathrm{mm}$ & 166 & 50 & 27 \\
RF duty cycle & $\%$ & 1 & 1 & 0.5 \\
\hline FEL Parameters & & & $<27$ & $50-100$ \\
\hline typical saturation length & $\mathrm{m}$ & $<14$ & 27.3 & $5-7$ \\
undulator period length & $\mathrm{mm}$ & 27.3 & 1.27 & $4.1-5.6$ \\
undulator K-parameter (planar) & & 1.27 & $>6$ & $0.1-10$ \\
photon wavelength range (1st harmon.) & $\mathrm{nm}$ & $>42$ & 3 & $60-280$ \\
peak photon beam power & $\mathrm{GW}$ & 0.2 & 40 & $10-500$ \\
number of photons per bunch & $10^{12}$ & 100 & 30 & 1 \\
typical photon beam divergence (rms) & $\mu \mathrm{rad}$ & 100 & 55 & 20 \\
typical photon beam diameter (rms) & $\mu \mathrm{m}$ & 70 & & \\
\hline
\end{tabular}

\section{ACCELERATION}

Each of the superconducting acceleration modules of the TESLA Test Facility provides a nominal acceleration of $120 \mathrm{MeV}$. For a more detailed desciption and the status, see Ref. [6]. With respect to FEL performance, a specific issue are the wakefields, because even for TESLA with its big cavity volume, the energy distribution inside each bunch is distorted or even dominated by longitudinal wakefields if the bunches are compressed to $50 \mu \mathrm{m}$ rms bunchlength (phase 2). Analytical and numerical investigations have shown that the longitudinal wake potential accumulated over one TESLA module is close to the analytic solution of a point charge in an infinitely long periodic structure [18], see Fig. 6.

\section{UNDULATOR}

The undulator is the most prominent FEL specific component. It has two functions: It has to provide the sinusoidal magnetic field so that the FEL process can take place, and, in order to keep the beam-size small over the whole undulator length, the undulator has to be combined with a superimposed periodic quadrupole lattice. The design for the TTF FEL is based on a planar hybrid permanent magnet undulator [19], see Table 1. It will be fabricated in modules $4.5 \mathrm{~m}$ long each, see Fig. 7. Between these modules and inside the $12 \mathrm{~mm}$ undulator gap a number of high resolution beam position monitors will be placed to ensure that the overlap between electron beam and photon beam stays within the specified tolerance of approx. $10 \mu \mathrm{m}$ [20]. The undulator is using four additional magnets per halfperiod to provide focusing, see Fig. 8 . A $220 \mathrm{~mm}$ long prototype

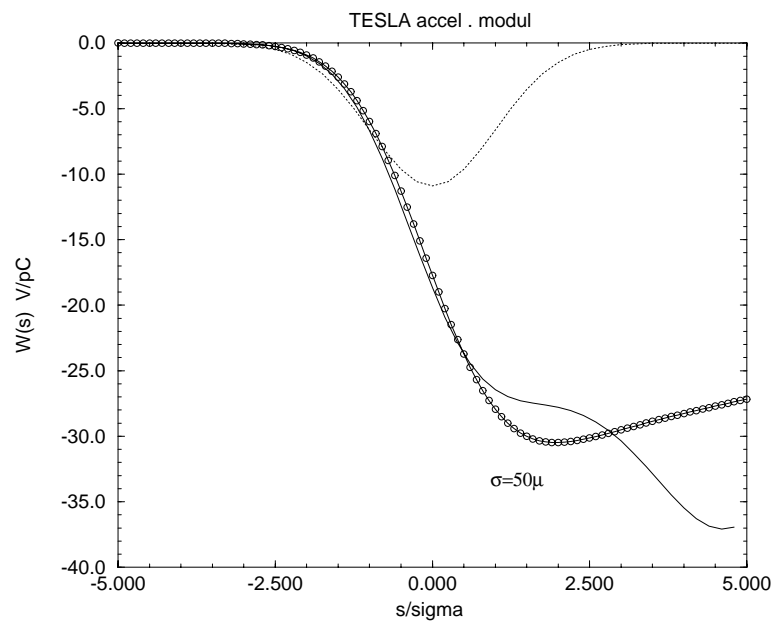

Figure 6: Wake potentials in the 8th cavity of a TESLA cryo-module for an rms bunch length of $0.05 \mathrm{~mm}$ and analytical approximation (circles). From Ref. [18].

has been built and tested, and two $1 \mathrm{~m}$ long prototypes are presently under construction.

\section{PHOTON BEAM PROPERTIES}

The prediction of photon beam parameters of short wavelength FELs is based on both an elaborate theory and various computer codes (for references, see [7] - [10]). Table 1 contains only few numbers on the expected FEL performance. The values quoted should be used as a guideline only, since there is no experimental experience yet in this wavelength regime. There is a radiation property that is specific to SASE FELs called superradiant spik- 


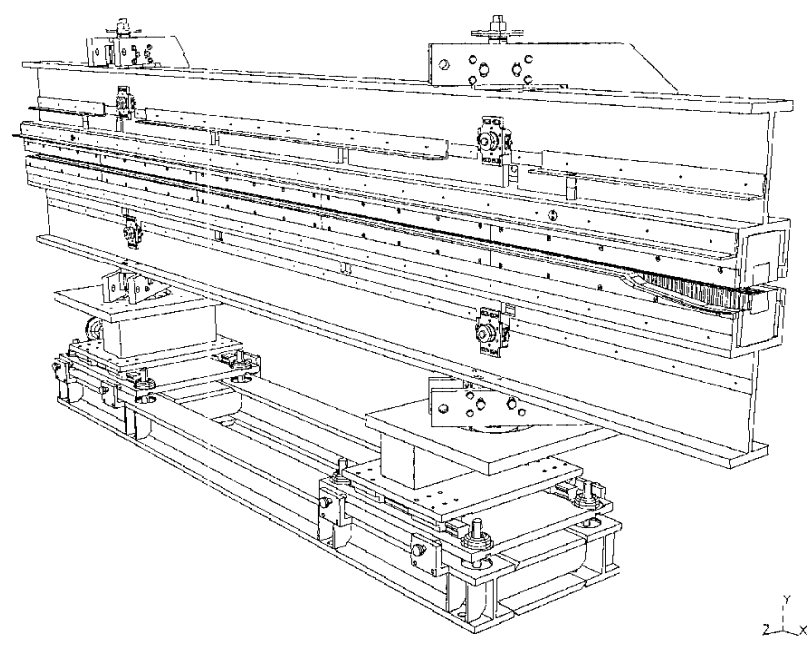

Figure 7: 3-D view of one undulator module for the VUVFEL at the TTF. From Ref. [9].

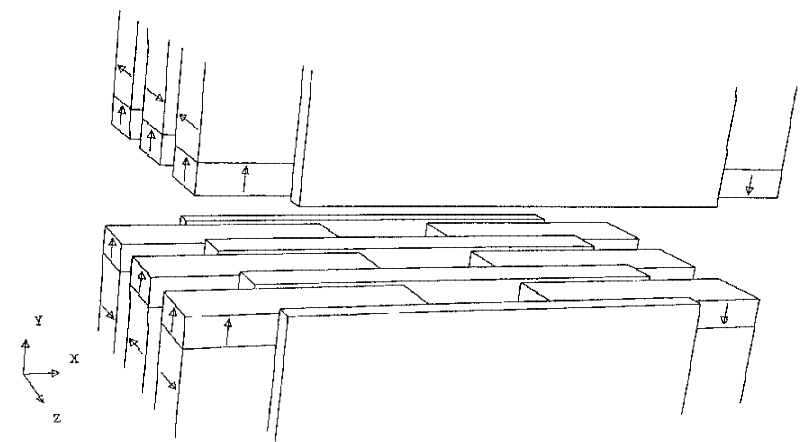

Figure 8: Perspective view of $1 \frac{1}{2}$ periods of the TTF FEL undulator which combines undulator field and strong focusing. From Ref. [19]. The small pole pieces with magnetization perpendicular to the gap plane provide the focusing field.

ing [21]. Due to the statistical character of the start-up from noise, the FEL radiation intensity is not homogeneously distributed along the pulse length but concentrated within ultrashort pulses as long as the cooperation length. This length is typically about 1000 times the photon wavelength, i.e. much shorter than the bunch length. There is no phase correlation between these spikes, so the spike length reflects the longitudinal coherence of the radiation. The photon statistics has been shown to be that of completely chaotic polarized radiation [22]. A two-stage FEL scheme has been devised to considerably reduce the bandwidth [23]. In Figs. 2, 3 its performance is referred to by the index $(\mathrm{M})$.

\section{REFERENCES}

[1] J.M.J. Madey, Stimulated Emission of Bremsstrahlung in a Periodic Magnetic Field, J. Appl. Phys. 42 1906, 1971

[2] A. M. Kondratenko, E. L. Saldin, Generation of Coherent
Radiation by a Relativistic Electron Beam in an Ondulator, Part. Accelerators, 10, 207, 1980.

[3] R. Bonifacio, C. Pellegrini, L.M. Narducci, Collective Instabilities and High-Gain Regime in a Free Electron Laser, Opt. Cummun. 50, No. 6, 373, 1984.

[4] R. Brinkmann, et al., An X-Ray FEL Laboratory as Part of a Linear Collider Design, Proc. 1996 FEL Conf., to be Published in Nucl. Instr. Meth. A, 1997.

[5] G. A. Loew (ed.), International Linear Collider Technical Review Committee Report, SLAC-R-95-471, 1995.

[6] B. Aune, Results from the DESY TESLA Test Facility, this conference

[7] T. Åberg, et al., A VUV Free-Electron Laser at the TESLA Test Facility at DESY, Conceptual Design Report, DESY Print TESLA-FEL 95-03, 1995.

[8] H. Winick, et al., A 2-nm - 4-nm Linac Coherent Light Source (LCLS) Using the SLAC Linac. SLAC-PUB-6185, May 1993. 3pp. Presented at 1993 Particle Accelerator Conference (PAC 93), Washington, DC, 17-20 May 1993.

[9] R. Brinkmann, G. Materlik, J. Rossbach, A. Wagner (eds.), Conceptual Design of a $500 \mathrm{GeV}$ e+e- Linear Collider with Integrated X-ray Laser Facility, DESY 1997-048 and ECFA 1997-182

[10] See e.g.: W.B. Colson, C. Pellegrini and R. Renieri (eds.), Laser Handbook, Vol.6, North-Holland, 1990

[11] J. Fraser and R. Sheffield, NIM A 250 71, 1986

[12] J.E. Clendenin, Proc. 18th Linac Conf., 298, Geneva, 1996

[13] I. Will, P. Nickles, W. Sandner, A Laser System for the TESLA Photo-Injector, internal design study, Max-BornInstitut, Berlin 1994.

[14] Ya. S. Derbenev, et al., DESY Print TESLA-FEL 95-05, 1995.

[15] E. L. Saldin, E. A. Schneidmiller, M. V. Yurkov, DESY Print TESLA-FEL 96-14, 1996.

[16] M. Dohlus, T. Limberg, Wake Fields of a Bunch on a General Trajectory Due to Coherent Synchrotron Radiation, this conference

[17] R. Tatchyn, et al. , Proc. PAC93, Washington, 1993.

[18] A.N.Novokhatski and A.Mosnier, Short Bunch Wake Potentials for a Chain of TESLA cavities, DAPNIA/SEA-96-08, 1996.

[19] J. Pflüger, Y. M. Nikitina, B. Faatz, T. Teichmann, The undulator system for the VUV-FEL at the TESLA Test Facility, Proc. 1996 Intl. FEL Conference, Rome, 1996, to be published.

[20] B. Faatz, J. Pflüger, Y.M. Nikitina, Study of the Undulator Specification for the VUV-FEL at the TESLA Test Facility, Proc. 1996 Intl. FEL Conference, Rome, 1996, to be published.

[21] R. Bonifacio, et al., Phys. Rev. Lett. 73 70, 1994.

[22] E. L. Saldin, E. A. Schneidmiller, M. V. Yurkov, DESY Print TESLA-FEL 97-02, 1997.

[23] J. Feldhaus, et al., Possible Application of X-ray Optical Elements for Reducing the Spectral Bandwidth of an $X$-ray SASE FEL, Proc. 1996 Intl. FEL Conference, Rome, 1996, to be published. 